\title{
Sleep Disturbances in Anorexia Nervosa: How Can They Be Explained?
}

\author{
Francisca Padez Vieira ${ }^{1 *}$, Pedro Afonso ${ }^{2}$
}

1 Hospital Dona Estefânia, Faculdade de Medicina da Universidade de Lisboa

2 Hospital Dona Estefânia, Centro Hospitalar e Universitário de Lisboa Central, EPE., Lisbon, Portugal

\section{ABSTRACT}

Patients with anorexia nervosa (AN) frequently complain of insomnia and poor sleep quality. Some studies report changes in sleep self-reports and in several polysomnography parameters. These studies suggest that patients with AN and concomitant sleep disturbances have a poorer quality of life, are more predisposed to the appearance of comorbidities and have a poor prognosis of AN. The presence of sleep problems appears to influence the course and prognosis of AN. This article intends to briefly discuss the possible impact of sleep disturbances in AN and describe potential pathophysiological mechanisms involved.

Keywords: Eating disorders, Polysomnography, Insomnia

Sleep is a complex physiological process, during which several vital physiologic functions occur, such as memory formation, mood and emotional regulation, neurocognitive performance, energy preservation and metabolic restoration. (National Sleep Foundation, n.d.) The association between sleep disturbances and psychopathology and psychiatric disorders has been frequently described. (Abad \& Guilleminault, 2005; Benca, Obermeyer, Thisted, \& Gillin, 1992) This suggests that recognition and management of sleep problems is essential when addressing the treatment, outcomes and prevention of mental illness relapse. (Abad \& Guilleminault, 2005)

Various authors have described an association between eating disorders (ED) and sleep problems.

\footnotetext{
*Correspondence: franciscavieira@gmail.com, 351 913812445, Hospital Dona Estefânia, Faculdade de Medicina da Universidade de Lisboa, Portugal. Received: 07 November 2019 Accepted: 18 June 2020
}

\section{Sleep and Hypnosis Journal homepage: http://www.sleepandhypnosis.org} ISSN:1302-1192 (Print) 2458-9101 (Online)
(Padez-Vieira \& Afonso, 2016) Very few studies have focused on the prevalence of sleep disturbances in anorexia nervosa (AN). Sleep disturbances have been reported in about half of the patients with eating disorders. (Kim et al., 2010) Not only patients' self-reports, but also families and doctors, describe that insomnia is frequently a complain of these patients. (Crisp, Stonehill, \& Fenton, 1971; Lombardo, Battagliese, Venezia, \& Salvemini, 2015)

Scarce data on self-reported sleep in AN suggest that gravely underweight patients describe poor sleep quality, difficulties in falling asleep, interrupted sleep, early morning waking or reduced total sleep time. (Crisp et al., 1971; Kim et al., 2010; Padez-Vieira \& Afonso, 2016; G Pieters, Theys, Vandereycken, Leroy, \& Peuskens, 2004)

Studies on objective sleep in AN have reported equivocal results. (Padez-Vieira \& Afonso, 2016) The results of studies using polygraphically monitored sleep and performed on individuals with AN are described in table 1 . Attempts to identify changes in the sleep architecture of patients with ED have so far led to conflicting results. (Lauer \& Krieg, 2004; Padez-Vieira \& Afonso, 2016) 
Table 1: Sleep in Anorexia Nervosa: Laboratory Polysomnographic FINDINGS

\begin{tabular}{|l|}
\hline Sleep variable \\
\hline$\downarrow$ Sleep efficiency \\
\hline$\uparrow$ REM latency \\
\hline$\downarrow$ REM latency \\
\hline$\uparrow$ REM density \\
\hline$\downarrow$ REM density \\
\hline$\downarrow$ Total sleep time \\
\hline$\downarrow$ SWS \\
\hline
\end{tabular}

Notes: Adapted from Veira \& Afonso, $2017^{4}$

Abbreviations: REM, rapid eye movement, SWS, slow wave sleep

Published data remains scarce, controversial and even contradictory in several aspects, with inconsistencies that may be due to different limitations of the studies. (Padez-Vieira \& Afonso, 2016)

These sleep disturbances have an impact on well-being, function and quality of life and may negatively affect the outcomes, the course and the prognosis of the disease. (Kim et al., 2010; Lombardo et al., 2015)

The possible causes of these sleep disturbances observed in anorexia nervosa are not clarified yet. There are at least four main possibilities, which are not mutually exclusive. One possible explanation is that the impairment in slow wave sleep in patients with AN seems to be due to psychiatric comorbidity. A second hypothesis is that sleep disturbances may depend on the neurologic changes that result from the state of malnutrition. (Nobili et al., 2004) Other possibility involved might be that sleep disturbances represent a "transdiagnostic" process, being common across different psychiatric disorders. (Fairburn, Cooper, \& Shafran, 2003; Harvey, 2008) This transdiagnostic perspective argues that perhaps disorders co-occur because they share common mechanisms (e.g., sleep disturbances). (Harvey 2008) Finally, some neurological have been identified as involved in both sleep and eating regulation and might be possibly implicated in both sleep and eating disorders. (Ohno \& Sakurai, 2008)

It is widely well-known that AN usually presents with multiple comorbidities. Depression, anxiety, and obsessive-compulsive disorder are some of the psychiatric disorders that often are comorbid in patients with AN. (American Psychiatric Association, 2013) The rates vary between studies. (Godart, Perdereau, Jeammet, \& Flament, 2005) All of these disorders may also interfere with sleep. Given the frequent association between sleep disorders and AN, it would be important to further investigate the contribution of $\mathrm{AN}$ to these sleep disorders, controlling for comorbidity.

A strong relationship between the state of nutrition and slow wave sleep (SWS) integrity seems to be supported by clinical data. (Levy, Dixon, \& Schmidt, 1988) A correlation between slow wave activity (SWA) reduction and the patient's body mass index (BMI) was reported. (Nobili et al., 1999) This correlation could be pointed out as an indirect indicator of the organic severity of this pathology. It suggests that the level of emaciation could (at least partially) result in the reduction of SWA. (Nobili et al., 2004)

After weight recovery, AN patients have the subjective experience of longer sleep duration and better sleep quality. (Lauer \& Krieg, 2004; Guido Pieters, Theys, Vandereycken, Leroy, \& Peuskens, 2004) The experimental induction of hunger in laboratory setting produces increased nocturnal wakefulness and reduced slow wave sleep. (Lombardo et al., 2015; Ohno \& Sakurai, 2008) In studies with male subjects subjected to severe and extended dietary restriction, sleep disruption has been described, with severe implications in physical and psychological health. (Keys, Brožek, Henschel, Mickelsen, \& Taylor, 1950) Prolonged food deprivation in animals has resulted in marked fragmentation of sleep and reduced amounts of SWS. (Lauer \& Krieg, 2004) The sleep disruptive effect of hunger is coherent with results of polysomnographic studies that indicate that hunger leads to reduced sleep efficiency and increased wake time in restricting type AN when compared with control groups. (Lauer \& Krieg, 2004; Lombardo et al., 2015)

On the other hand, there is evidence that weight recovery leads to hypersomnia. (Evans, 1983; Padez-Vieira \& Afonso, 2016) The induction of satiety appears to lead to an increase in sleepiness and a subsequent 'deepening' in sleep. (Lauer \& Krieg, 2004) In sleep studies, the consumption of food and 
the experimental use of gut-peptides and leptin elicit an increase in sleepiness, reduce sleep latency and enhance SWS. (Lauer \& Krieg, 2004)

Sleep disturbance is comorbid with many psychiatric disorders (Benca et al., 1992). It has been suggested that sleep disturbances are aetiologically linked to different forms of psychopathology through its reciprocal relationship with emotion regulation and shared neurobiological substrates. (Harvey, Murray, Chandler, \& Soehner, 2011) This consideration of sleep disturbances as a transdiagnostic risk factor has been purposed on the basis of: the high rates of co-occurrence between sleep disturbances and psychiatric disorders; the fact that the vast majority of the lifetime disorders are comorbid disorders, suggesting that perhaps disorders co-occur because they share common mechanisms (e.g., insomnia); the evidence across a variety of disorders that sleep disturbances contribute to the onset, relapse, and maintenance of psychopathology; and the transdiagnostic applicability of a bidirectional sleep-and-mood model. (Harvey, 2008; Harvey et al., 2011)

Finally, it has been demonstrated that the orexin system regulates sleep and wakefulness through interactions with systems that regulate emotion, reward, and energy homeostasis. In fact, orexin receptors play an important role in regulating sleep/awake states. (Chemelli et al., 1999; L Lin et al., 1999; Mieda et al., 2004) At the same time, this system is also related to the limbic system, which regulates emotional responses, the reward system, and hypothalamic mechanisms that stimulate feeding behavior. (Ohno \& Sakurai, 2008) Orexin neurons are placed in the lateral hypothalamic area, facilitating the link between the limbic system, energy homeostasis, and brain stem monoaminergic/cholinergic neurons. (Ohno \& Sakurai, 2008) The responsiveness of orexin neurons to peripheral metabolic cues, such as leptin and glucose, suggests that these cells might play an important role as a sensor of the metabol- ic status. (Ohno \& Sakurai, 2008; Yamanaka et al., 2003) These findings indicate that orexin neurons provide a crucial link between arousal, emotion, energy balance and reward systems, and act as a regulator that maintains proper vigilance states according to inner and outer environments of our bodies. (Ohno \& Sakurai, 2008) They also suggest that this identified neurological centers are involved in both sleep and eating regulation and might be possibly implicated in both sleep and eating disorders.

More studies are needed to better understand and scientifically substantiate if there is an association between sleep disturbances and AN, to characterize sleep more clearly in this population, and to understand the mechanisms that can explain these sleep disturbances.

If comorbidity is present, it is essential to treat the comorbid disorder, as it can lead to sleep disturbances and affect the disease prognosis.

As previously mentioned, sleep disturbances have an impact on the quality of life of patients. (Strine \& Chapman, 2005) Patients with AN and sleep problems, have a higher predisposition to appearance of mood disorders, more severe symptomatology and resistance to treatment. (Kim et al., 2010; Lombardo et al., 2015; Strine \& Chapman, 2005) So, these sleep disturbances may negatively affect the outcomes, the course and the prognosis of the illness.

A wider knowledge in this area is highly relevant to understand the prognosis and adherence to treatment if sleep disturbances are present, and also for the therapeutics and follow-up of these situations. The impact that sleep disturbances seem to have on the evolution and prognosis of the disease, justifies an assessment of the impact of weight gain on sleep quality and quality of life, as well as the impact of sleep disturbances in the severity of the symptomatology.

Conflict of interest: "The authors declare no conflict of interest”. 


\section{References}

Abad, V., \& Guilleminault, C. (2005). Sleep and Psychiatry. Dialogues in Clinical Neuroscience, 7(4), 291-303.

American Psychiatric Association. (2013). Diagnostic and Statistical Manual of Mental Disorders (5th ed.). Arlington, VA: American Psychiatric Press, Inc.

Benca, R., Obermeyer, W., Thisted, R., \& Gillin, J. (1992). Sleep and psychiatric disorders. A meta-analysis. Arch Gen Psychiatry, 49(8), 651-668.

Chemelli, R. M., Willie, J. T., Sinton, C. M., Elmquist, J. K., Scammell, T., Lee, C., ... Yanagisawa, M. (1999). Narcolepsy in orexin knockout mice: Molecular genetics of sleep regulation. Cell, 98(4), 437-451. https://doi.org/10.1016/Soo92-8674(oo)81973-X

Crisp, a H., Stonehill, E., \& Fenton, G. W. (1971). The relationship between sleep, nutrition and mood: a study of patients with anorexia nervosa. Postgraduate Medical Journal, 47(546), 207213. https://doi.org/10.1136/pgmj.47.546.207

Evans, F. (1983). Sleep, eating and weight disorders. In Goodstein (Ed.), Sleep, eating and weight disorders. (pp. 147-178). New York: Springer.

Fairburn, C. G., Cooper, Z., \& Shafran, R. (2003). Cognitive behaviour therapy for eating disorders: A "transdiagnostic" theory and treatment. Behaviour Research and Therapy, 41(5), 509-528. https://doi.org/10.1016/Sooo5-7967(02)0oo88-8

Godart, N., Perdereau, F., Jeammet, P., \& Flament, M. (2005). Comorbidity between eating disorders and mood disorders: review. Encephale, 31, 575-87.

Harvey, A. G. (2008). Insomnia, psychiatric disorders, and the transdiagnostic perspective. Current Directions in Psychological Science, 17(5), 299-303. https://doi.org/10.1111/j.14678721.2008.00594.x

Harvey, A. G., Murray, G., Chandler, R. A., \& Soehner, A. (2011). Sleep disturbance as transdiagnostic: Consideration of neurobiological mechanisms. Clinical Psychology Review, 31(2), 225235. https://doi.org/10.1016/j.cpr.2010.04.003

Keys, A., Brožek, J., Henschel, A., Mickelsen, O., \& Taylor, H. L. (1950). The Biology of Human Starvation (Vols. 1-2). Minneapolis: University of Minnesota Press.

Kim, K., Jung, Y., Shin, M., Namkoong, K., Kim, J., \& Lee, J. (2010). Sleep disturbance in women with eating disorder: prevalence and clinical characteristics. Psychiatry Res, 176(1), 88-90.

Lin, L. , Faraco, J., Li, R., Kadotani, H., Rogers, W., Lin, X., Qiu, X., de Jong, P.J., Nishino, S. (1999). The Sleep Disorder Canine Narcolepsy Is Caused by a Mutation in the Hypocretin (Orexin). Cell, 98(August), 365-376. https://doi. org/10.1145/2636240.2636876
Lauer, C., \& Krieg, J. (2004). Sleep in eating disorders. Sleep Medicine Reviews, 8(2), 109-118. https://doi.org/10.1016/S10870792(02)00122-3

Levy, A., Dixon, K., \& Schmidt, H. (1988). Sleep Architecture in Anorexia Nervosa and Bulimia. Biological Psychiatry, 23(1), 99-101.

Lombardo, C., Battagliese, G., Venezia, C., \& Salvemini, V. (2015). Persistence of poor sleep predicts the severity of the clinical condition after 6 months of standard treatment in patients with eating disorders. Eating Behaviors, 18(March 2013), 16-19. https://doi.org/10.1016/j.eatbeh.2015.03.003

Mieda, M., Willie, J. T., Hara, J., Sinton, C. M., Sakurai, T., \& Yanagisawa, M. (2004). Orexin peptides prevent cataplexy. 101(13), 4649-4654.

National Sleep Foundation. (n.d.). What Happens When You Sleep? Retrieved July 2, 2019, from https://www.sleepfoundation.org/ articles/what-happens-when-you-sleep

Nobili, L., Baglietto, M., Beelke, M., Carli, F., Comite, R., Fiocchi, I., ... Ferrillo, F. (2004). Impairment of the Production of Delta Sleep in Anorectic Adolescents. Biological Psychiatry, 27(8), 1553-1559.

Nobili, L., Baglietto, M., De Carli, F., Savoini, M., Schiavi, G., Zanotto, E., ... De Negri, M. (1999). A quantified analysis of sleep electroencephalography in anorectic adolescents. Biological Psychiatry, 45(6), 771-775. https://doi.org/10.1016/Sooo63223(98)00107-3

Ohno, K., \& Sakurai, T. (2008). Orexin neuronal circuitry: Role in the regulation of sleep and wakefulness. Frontiers in Neuroendocrinology, 29(1), 70-87. https://doi.org/10.1016/j.yfrne.2007.08.001

Padez-Vieira, F., \& Afonso, P. (2016). Sleep disturbances in anorexia nervosa. Advances in Eating Disorders, 4(2), 176-188. https:// doi.org/10.108o/21662630.2016.1175958

Pieters, G, Theys, P., Vandereycken, W., Leroy, B., \& Peuskens, J. (2004). Sleep Variables in Anorexia Nervosa: Evolution with Weight Restoration. International Journal of Eating Disorders, 35(3), 342-347. https://doi.org/10.1002/eat.10256

Strine, T., \& Chapman, D. (2005). Associations of frequent sleep insufficiency with health-related quality of life and health behaviors. Sleep Med., 6(1), 23-27.

Yamanaka, A., Beuckmann, C. T., Willie, J. T., Hara, J., Tsujino, N., Mieda, M., ... Sakurai, T. (2003). Hypothalamic orexin neurons regulate arousal according to energy balance in mice. Neuron, 38(5), 701-713. https://doi.org/10.1016/So8966273(03)00331-3 